\title{
Measuring AGN Feedback Parameters From Seyfert Galaxy Outflows
}

\author{
F. Müller-Sánchez ${ }^{1}$, M. Malkan¹, E. K. S. Hicks ${ }^{2}$, and R. I. Davies ${ }^{3}$ \\ ${ }^{1}$ Department of Physics and Astronomy, University of California, Los Angeles, USA \\ ${ }^{2}$ University of Washington, Seattle, USA \\ ${ }^{3}$ Max-Planck-Institut für extraterrestrische Physik, Garching, Germany
}

\begin{abstract}
We present results of an on-going program to measure AGN feedback in Seyfert galaxies using integral-field spectroscopy and adaptive optics at Keck Observatory and VLT. Our integral-field observations are revealing AGN-driven outflows of ionized gas in Seyfert galaxies. By resolving the inner 10-40 parsecs, we are successfully modeling them as biconical structures, in which the ionized gas first accelerates and then decelerates. The model parameters provide crucial information on the orientation, geometry and kinematics of the outflows, which is used to estimate mechanical feedback from the AGN: mass and kinetic energy transferred to the interstellar medium. Mass outflow rates can be $10^{2}-10^{4}$ times greater than accretion rates, but in some cases, they are comparable to the estimated inflow rates to the central $10-25 \mathrm{pc}$, suggesting that the outflows may remove a considerable amount of the infalling gas before it reaches the accretion disk. In half of the AGN measured so far, the kinetic energy of the outflows appears sufficient to provide the eagerly-sought AGN feedback invoked to explain fundamental galaxy properties such as the $M_{\mathrm{BH}}-\sigma^{*}$ relation $\left(0.5-5 \% L_{\mathrm{bol}}\right)$. The other AGN, which lack powerful outflows, also have weaker and more compact radio jets.
\end{abstract}

Keywords. galaxies: active - galaxies: kinematics and dynamics — galaxies: nuclei - galaxies: Seyfert — galaxies: evolution — infrared: galaxies

\section{Introduction}

The discovery of a number of black hole (BH)-galaxy relations, such as the $M_{\mathrm{BH}}-$ $\sigma^{*}$ relation (e.g. Gebhardt et al. 2000, Ferrarese \& Merritt 2000), has shown that the growth of supermassive BHs is closely related to the evolution of galaxies. Mechanical feedback from Active Galactic Nuclei (AGN)-driven winds is commonly invoked as the key mediator between the coevolution of BHs and their host galaxies (e.g. Silk \& Rees 1998, di Matteo et al. 2005, Springel et al. 2005, Hopkins \& Elvis 2010): AGN outflows provide energy into the interstellar medium (ISM), controlling the star formation and accretion processes, thus regulating BH and bulge growth. Although AGN feedback is useful for various purposes, there is little observational data to date to constrain its real impact in the evolution of galaxies.

The narrow-line and coronal-line regions (NLR and CLR) in AGN are crucial in attempts to understand the interaction between the nuclear engine and the ISM, since they are the largest observable structures in the UV, optical and near-IR $(\sim 500 \mathrm{pc}$ in Seyfert galaxies) that are directly affected by the ionizing radiation and dynamical forces from the AGN. The study of high-ionization lines (or coronal lines) is particularly important because they are unique tracers of AGN activity. In contrast to [O III] or $\mathrm{H} \alpha$ which are commonly used to study the NLR, the coronal lines are free of contributions from star formation. Therefore, the CLR provides direct insights into the structures and dynamical forces associated with the AGN, such as ionized gas in radiatively accelerated outflows. 
An ideal technique for measuring AGN feedback is to map the kinematics of the NLR/CLR in Seyfert galaxies (which accrete at considerable Eddington ratios and are luminous enough to be considered local counterparts of quasars, yet close enough to study at high angular resolution) using Adaptive Optics (AO) assisted integral-field spectroscopy. Over the past eight years we have made an integral-field spectroscopic survey of Seyfert galaxies, using AO and the instruments SINFONI on the VLT and OSIRIS on the Keck Observatory to probe the spatial distribution and kinematics of the NLR and CLR at spatial resolutions better than $0.1^{\prime \prime}$. The $\operatorname{Br} \gamma$ recombination line of hydrogen at $2.16 \mu \mathrm{m}$ and the [SiVI] emission line at $1.96 \mu \mathrm{m}$ are used as tracers of the NLR and CLR, respectively. Our initial work with seven Seyfert galaxies has shown that we can model the NLR and CLR kinematics as a combination of rotating and outflowing components (Müller-Sánchez et al. 2011). In this contribution we report updated results of our ongoing program to measure AGN feedback from Seyfert galaxy outflows, including new data for one Seyfert 2 galaxy (NGC 3081).

\section{Modeling the Kinematics of the NLR and CLR}

Throughout our analysis we are interpreting our observations in the context of kinematic models of the NLR. Specifically, we are comparing our 2D kinematic data with models comprising biconical outflows superimposed on disk rotation. A simple biconical geometry has been adopted to match the shape of the NLR as suggested by popular unification models of AGN (e.g. Antonucci 1993, Urry \& Padovani 1995). The bicone model consists of two symmetrical hollow cones having interior and exterior walls with apices coincident with a central point source. Material flows inside the walls of the bicone, i.e. each point of the bicone corresponds to a velocity vector. The simplest velocity law which reproduces the observations is radial linear acceleration followed by radial linear deceleration. To match the amplitudes of the measured blueshifted and redshifted velocities, the bicone can be tilted and rotated in space. Therefore, the bicone model has seven free parameters: inner and outer half-opening angles $\left(\theta_{\text {in }}\right.$ and $\left.\theta_{\text {out }}\right)$, position angle and inclination of the bicone's major axis (P.A.cone and $\left.i_{\text {cone }}\right)$, height of each cone $\left(z_{\max }\right)$, turnover radius at which the deceleration phase starts $\left(r_{t}\right)$ and maximum velocity $\left(v_{\max }\right)$. The disk model has three free parameters: magnitude of the circular velocity $\left(v_{c}\right)$, position angle of the major axis of the disk (P.A.disk), and inclination of the disk $\left(i_{\text {disk }}\right)$. As can be seen in Fig. 1 for the case of NGC 3081, our kinematic models of biconical outflow plus rotation provide a good match to the observed velocity fields of the NLR/CLR (see also Müller-Sánchez et al. 2011).

\section{Implications for AGN and Galaxy Evolution}

\subsection{Geometry of the outflows}

The biconical structures have full opening angles $\left(2 \theta_{\text {out }}\right)$ in the range $54-114^{\circ}$. We find that this parameter is anticorrelated with the molecular gas mass in $r<30 \mathrm{pc}$, which can be understood if the accumulation of gas clouds toward the nucleus increases the collimation of the outflows.

The typical distance at which the outflows reach $v_{\max }$ is $r_{t}=210 \mathrm{pc}$ (with values ranging from $130-330 \mathrm{pc}$, except in NGC 2992 where $r_{t}=900 \mathrm{pc}$ ). Even taking into account the deceleration phase (and a typical distance $z_{\max }=2 r_{t}$ at which the outflow velocity is zero), we conclude that AGN-driven outflows in Seyfert galaxies extend to scales of hundreds of parsecs. For the case of NGC 2992, we interpret its geometrical 

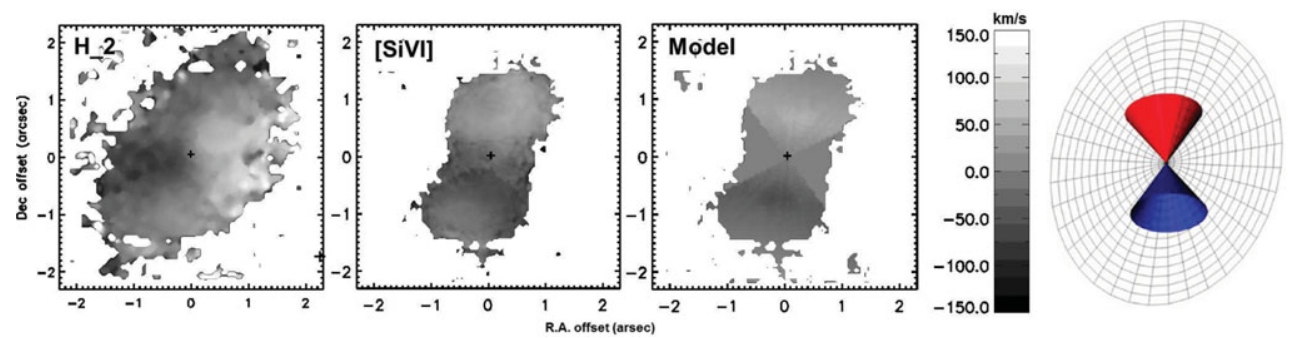

Figure 1. The bipolar outflow in the Seyfert 2 galaxy NGC 3081. The left two panels show the velocity fields of $\mathrm{H}_{2}$ and [SiVI] emission in the central $320 \times 320 \mathrm{pc}$ of the galaxy. The kinematics of the highly-ionized gas shows a velocity gradient in a different direction to the rotation of the molecular gas, indicative of an outflow of $>200 \mathrm{~km} \mathrm{~s}^{-1}$. The right two panels show the best-fit biconical model to the [SiVI] velocities and the geometric model of the NLR/CLR and the inner galactic disk. This geometry suggests an outflow axis nearly perpendicular to the line-of-sight -consistent with unified schemes for Seyfert $2 \mathrm{~s}$.

parameters (the largest opening angle and $r_{t}$ ) as evidence for a hybrid origin of the outflow (stellar and the AGN). Similar kpc-scale outflows are observed in nearby starburst galaxies (M82: Westmoquette et al. 2009, NGC 253: Westmoquette et al. 2011).

Finally, our 3D models of the geometry of the bicones provide a direct test of the hypothesis that orientation is the primary difference between type 1 and type 2 AGN (e.g. Antonucci 1993). Of the seven Seyferts which have been modeled in this way (MüllerSánchez et al. 2011, 2012), five are type 1. The Seyfert 2s (NGC 1068 and NGC 3081) are found to be nearly edge-on, consistent with unified schemes (Fig. 1). However, contrary to the simple expectations of face-on Seyfert 1s, we find that our sample of five are considerably inclined, with one nearly edge-on (NGC 3783). This would require a modification to simple unification, such as a highly clumpy torus.

\subsection{Strength of the outflows}

From the measured geometrical parameters and velocities we estimate mass outflow rates and the kinetic power of the outflows $\left(\dot{E}_{\text {out }}\right)$. The resulting mass outflow rates are in the range $1-10 \mathrm{M}_{\odot} \mathrm{yr}^{-1}$ and the estimated kinetic powers between $0.05-5 \times 10^{42} \mathrm{erg}$ $\mathrm{s}^{-1}$. These are $10^{2}-10^{4}$ times smaller than the AGN's bolometric luminosities. In half of the observed AGN (NGC 1068, NGC 2992, NGC 3081 and NGC 4151) the kinetic power of the outflow is of the order of the power required by feedback models to explain fundamental galaxy properties $\left(\sim 0.005-0.05 L_{\mathrm{bol}}\right.$, di Matteo et al. 2005 , Hopkins \& Elvis 2010). While in these objects the radio jet is extended and clearly interacting with the ISM, in the other half of AGN - in which the outflow power is at least one order of magnitude less than predicted in feedback models - the radio jet is weak and compact. This suggests a link between jet power and outflow power (Fig. 2a).

We find no correlation between AGN luminosity (and therefore current accretion rate) and $\dot{E}_{\text {out }}$. In fact, one of the objects in our sample is a low luminosity AGN with a relatively strong outflow: NGC 3081. This is a Seyfert 2 galaxy with a bolometric luminosity of $\sim 15 \times 10^{42} \mathrm{erg} \mathrm{s}^{-1}$ (Nicastro et al. 2003), a factor of $\sim 4$ below the rest of Seyfert galaxies in our sample. However, its ratio of $\dot{E}_{\text {out }} / L_{\text {bol }} \sim 0.005$ is in the regime of values required by AGN feedback models. These results suggest that the outflow is probably not a single wind originating in the AGN, but is actually a product of the interaction of an already accelerated wind with its ambient medium. 

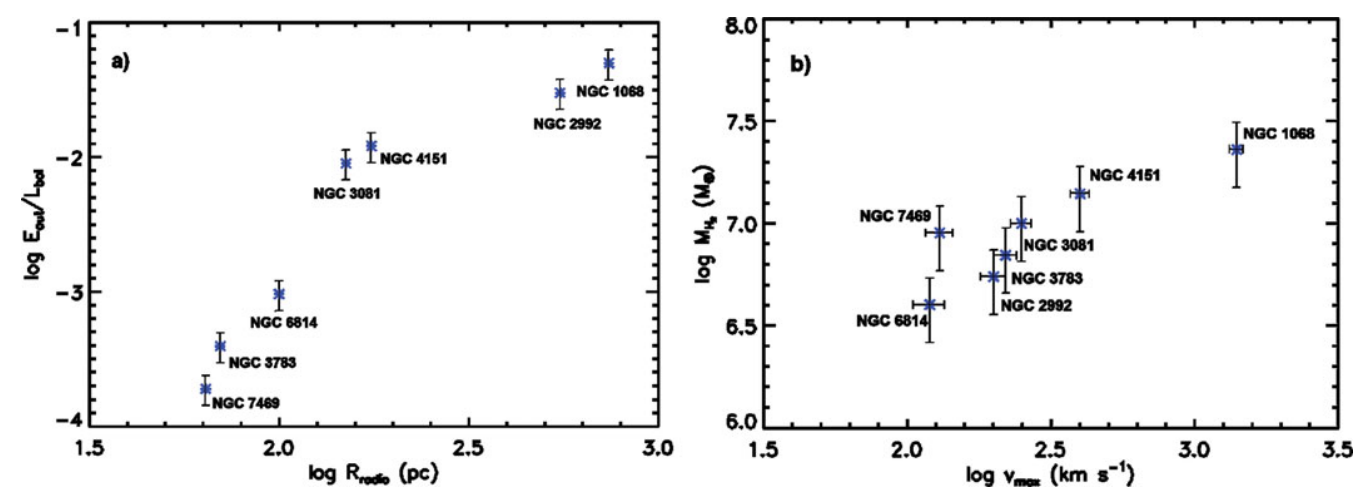

Figure 2. Graphs showing the correlations found between a) the size of the radio emission associated with the AGN $\left(\mathrm{R}_{\text {radio }}\right)$ and the ratio of outflow power to accretion power $\left(\dot{E}_{\text {out }} / L_{\mathrm{bol}}\right)$, and $\mathrm{b})$ the maximum velocity of the outflow $\left(v_{\max }\right)$ and the molecular gas mass $\left(\mathrm{M}_{H_{2}}\right)$ in the central $r<30$ pc (data from Müller-Sánchez et al. 2011, 2012).

\subsection{Feeding versus Feedback}

With the few objects analyzed to date, there are hints of a correlation between $v_{\max }$ and the central gas mass, which can be understood if larger gas masses constrict the outflow more (Fig. 2b). In addition, the inferred outflow rates can be 2 or more orders of magnitude greater than the required accretion rates to fuel the AGN at its current luminosity. However, in three galaxies (NGC 1068, NGC 3081 and NGC 4151), they are comparable to the estimated inflow rates to the central $10-25 \mathrm{pc}$ (Storchi-Bergmann et al. 2010, Müller-Sánchez et al. 2009, 2012). These three facts point to an interaction between the outflow and the ISM. The infalling gas accumulates on scales of a few tens of parsecs and the outflows prevent the majority of the gas from reaching the accretion disk (disrupting the conditions necessary for star formation as well).

\section{References}

Antonucci, R. 1993, ARAA, 31, 473

di Matteo, T., Springel, V. \& Hernquist, L. 2005, Nature, 433, 604

Ferrarese, L. \& Merritt, D. 2000, ApJ, 539, L9

Gebhardt, K., Bender, R., Bower, G., Dressler, A., Faber, S. M., Filippenko, Alexei V., Green, R., Grillmair, C., Ho, L. C., \& Kormendy, J. 2000, ApJ, 539, 13

Hopkins, P. F. \& Elvis, M. 2010, MNRAS, 401, 7

Müller-Sánchez, F., Davies, R. I., Genzel, R., Tacconi, L. J., Eisenhauer, F., Hicks, E. K. S., Friedrich, S., \& Sternberg, A. 2009, ApJ, 691, 749

Müller-Sánchez, F., Prieto, M. A., Hicks, E. K. S., Vives-Arias, H., Davies, R. I., Malkan, M., Tacconi, L. J., \& Genzel, R. 2011, ApJ, 739, 69

Müller-Sánchez, F., Malkan, M., \& Hicks, E. K. S. 2012, ApJ, in prep.

Nicastro, F., Martocchia, A., \& and Matt, G. 2003, ApJ Letters, 589, 13

Silk, J., Rees M. J. 1998, A\&A, 331, L1

Springel, V., et al. 2005, MNRAS, 361, 776

Storchi-Bergmann, T., McGregor, P. J., Riffel, R. A., Simoes Lopes, R., Beck, T., \& Dopita, M. 2010, MNRAS, 394, 1148

Urry, C. M. \& Padovani, P. 1995, PASP, 107, 803

Westmoquette, M. S., Smith, L. J., Gallagher, J. S., Trancho, G., Bastian, N., \& Konstantopoulos, I. S. 2009, ApJ, 696, 192

Westmoquette, M. S., Smith, L. J., \& Gallagher, J. S. 2011, MNRAS, 414, 3719 